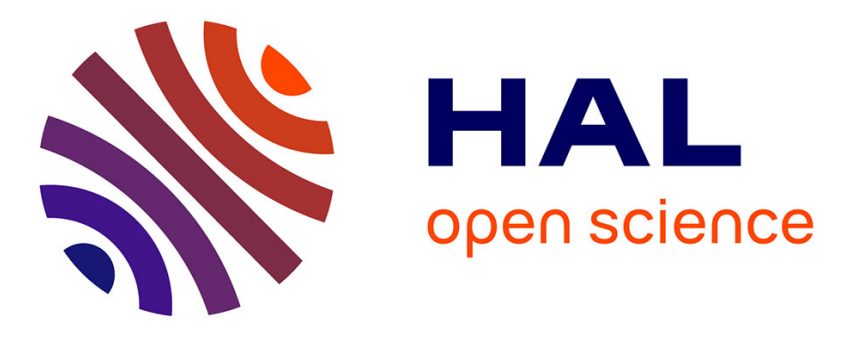

\title{
Les réalités migratoires au prisme de la ville
}

\author{
Marie Poinsot, Chowra Makaremi, Véronique Bontemps
}

\section{To cite this version:}

Marie Poinsot, Chowra Makaremi, Véronique Bontemps. Les réalités migratoires au prisme de la ville: Entretien avec Véronique Bontemps, anthropologue, chargée de recherche au CNRS et Chowra Makaremi, anthropologue et chercheuse au CNRS (IRIS-EHESS Paris). Hommes \& migrations, 2018, 2018/4 ( $\mathrm{n}^{\circ} 1323$ ), pp.31 - 37. 10.4000/hommesmigrations.7191 . hal-03038442

\section{HAL Id: hal-03038442 \\ https://hal.science/hal-03038442}

Submitted on 3 Dec 2020

HAL is a multi-disciplinary open access archive for the deposit and dissemination of scientific research documents, whether they are published or not. The documents may come from teaching and research institutions in France or abroad, or from public or private research centers.
L'archive ouverte pluridisciplinaire HAL, est destinée au dépôt et à la diffusion de documents scientifiques de niveau recherche, publiés ou non, émanant des établissements d'enseignement et de recherche français ou étrangers, des laboratoires publics ou privés. 


\section{LES RÉALITÉS MIGRATOIRES AU PRISME DE LA VILLE Entretien avec Véronique Bontemps, anthropologue, chargée de recherche au CNRS et Chowra Makaremi, anthropologue et chercheuse au CNRS (IRIS-EHESS Paris), Marie Poinsot}

Musée de l'histoire de l'immigration | «Hommes \& Migrations »

2018/4 $\mathrm{n}^{\circ} 1323$ | pages 31 à 37

ISSN 1142-852X

Article disponible en ligne à l'adresse:

https://www.cairn.info/revue-hommes-et-migrations-2018-4-page-31.htm

Distribution électronique Cairn.info pour Musée de l'histoire de l'immigration.

(C) Musée de l'histoire de l'immigration. Tous droits réservés pour tous pays.

La reproduction ou représentation de cet article, notamment par photocopie, n'est autorisée que dans les limites des conditions générales d'utilisation du site ou, le cas échéant, des conditions générales de la licence souscrite par votre établissement. Toute autre reproduction ou représentation, en tout ou partie, sous quelque forme et de quelque manière que ce soit, est interdite sauf accord préalable et écrit de l'éditeur, en dehors des cas prévus par la législation en vigueur en France. Il est précisé que son stockage dans une base de données est également interdit. 


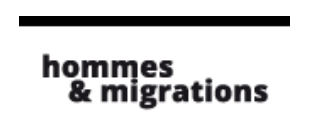

\section{Hommes \& migrations}

Revue française de référence sur les dynamiques

migratoires

1323 | 2018

Persona grata

\section{Les réalités migratoires au prisme de la ville}

Entretien avec Véronique Bontemps, anthropologue, chargée de recherche au CNRS et Chowra Makaremi, anthropologue et chercheuse au CNRS (IRIS-EHESS Paris),

\section{Marie Poinsot}

\section{(2) OpenEdition}

Journals

Édition électronique

URL : http://journals.openedition.org/hommesmigrations/7191

DOI : 10.4000/hommesmigrations.7191

ISSN : 2262-3353

Éditeur

Musée national de l'histoire de l'immigration

Édition imprimée

Date de publication : 1 octobre 2018

Pagination : 31-37

ISBN : 978-2-919040-42-1

ISSN : 1142-852X

Distribution électronique Cairn

\section{CAIRN INFO}

CHERCHER, REPÉRER, AVANCER

Référence électronique

Marie Poinsot, « Les réalités migratoires au prisme de la ville », Hommes \& migrations [En ligne], 1323 | 2018, mis en ligne le 01 octobre 2020, consulté le 14 mars 2019. URL : http://

journals.openedition.org/hommesmigrations/7191; DOI : 10.4000/hommesmigrations.7191 


\section{Les réalités migratoires au prisme de la ville}

Entretien avec

Véronique Bontemps,

anthropologue, chargée de recherche au CNRS

Chowra Makaremi,

anthropologue et chercheuse au CNRS (IRIS-EHESS Paris) ${ }^{1}$

réalisé par Marie Poinsot,

rédactrice en chef de la revue.

\section{H}

ommes \& Migrations: Quels sont les objectifs du programme de recherche Babels et les bienfaits scientifiques des échanges internationaux entre les différentes équipes? Le point de vue de la société d'accueil

Véronique Bontemps: Le programme Babels est une réponse à un appel à projet lancé par l'Agence nationale de la recherche dans le cadre d'un «Flash asile» en résonance avec ce que les médias avaient nommé «la crise migratoire» durant l'été 2015. Ce programme s'est constitué autour de Michel Agier pour saisir les spécificités de cette crise en la replaçant dans une histoire plus longue des migrations et à l'échelle européenne et au-delà, notamment au Moyen-Orient. Bien entendu, les recherches sur les migrations sont anciennes et très nombreuses. Les travaux rappellent que cette crise migratoire n'est pas nouvelle dans les formes des flux migratoires vers l'Europe et qu'elle s'inscrit dans le cadre d'une crise des politiques migratoires - expression que nous préférons à «crise migratoire». Les chiffres sur ces migrations montrent très bien qu'il n'y a pas eu d'explosion migratoire. Les travaux autour de l'Atlas des migrations, réactualisé par Catherine Wihtol de Wenden, l'équipe de Migrinter et Migreurope développent des recherches sur ces enjeux et ces deux dernières équipes collaborent avec le programme Babels.

Chowra Makaremi : Les disciplines mobilisées par le programme Babels sont multiples et complémentaires. Il y a des géographes, des anthropologues et des sociologues principalement qui menaient déjà des terrains sur le long court sur ces thématiques et qui ont mutualisé leurs résultats dans le programme. Ce travail est le fruit de la collaboration d'équipes travaillant sur des terrains très déterminants en regard de la géographie des migrations et au prisme des villes, comme sur Vintimille, Calais, etc. Le programme a plutôt cherché à mettre en relation des chercheurs en produisant de nouveaux réseaux. À l'échelle européenne, beaucoup de chercheurs ont travaillé sur leur propre pays, que ce soit en Italie, au Danemark, en Allemagne, en France ou à Istanbul. Le programme se fonde sur des échanges et des discussions entre chercheurs à partir des travaux déjà menés par chaque équipe sur son terrain.

1. Ces deux chercheuses ont contribué à l'ouvrage Entre accueil et rejet. Ce que les villes font aux migrants, éd. Le passager clandestin, coll. "Bibliothèque des Frontières », 2018 
V. B. : L'essentiel du programme consiste à mettre les équipes en réseaux et à instaurer un dialogue entre les chercheurs. C'est pour cela que les Ateliers d'anthropologie publique ont été mis en place et pensés comme des temps forts de discussion entre chercheurs sur les résultats, mais où sont aussi invités des personnalités extérieures à la recherche et des acteurs de terrain. D'ailleurs, l'ouvrage Entre accueil et rejet. Ce que les villes font aux migrants est le fruit d'un de ces ateliers de discussions sur le rôle des villes européennes dans l'accueil, ou non, des migrants dans un processus de co-production des savoirs grâce à la participation de toutes ces personnalités.

\section{L'essentiel du programme consiste à mettre les équipes en réseaux et à instaurer un dialogue entre les chercheurs}

C. M. : Cet ouvrage est une mise en écho de la manière dont les villes font réseaux sur le thème de l'accueil des migrants. Il rassemble, compare et analyse différentes expériences de politiques municipales dans ce domaine, non seulement en relation avec les politiques nationales ou régionales, mais aussi avec la manière dont les migrations fonctionnent également en réseaux, en reprenant les trajectoires des circulations, des nœuds et des points de passage et d'arrivée des migrants eux-mêmes en Europe. Et cela qualifie aussi la manière dont les chercheurs ont travaillé en réseaux. H\&M: Quel est l'intérêt d'aborder les migrations à l'échelle des villes et non à l'échelle nationale? Quels sont les apports d'une démarche locale et territorialisée?

C. $\mathbf{M}$. : Les recherches sur les migrations en Europe ne sont pas forcément établies à l'échelle nationale et avec le prisme analytique des États. La prégnance de l'échelle nationale tient plutôt aux tendances disciplinaires sur ces thématiques. Par exemple, l'anthropologie des frontières, qui est une thématique plus récente dans le domaine migratoire, observe les circulations au prisme des frontières nationales, des contrôles étatiques et de leurs entraves et elle ne s'intéresse pas vraiment à la ville. Les études qui prennent comme unité d'analyse les villes sont davantage mobilisées sur les dynamiques des migrations sur le long terme, en traitant des questions d'installation, d'insertion et de citoyenneté, dans le prolongement des travaux de Arjun Apaduraï dans les années 1990, des citizenship-studies, des études sur les communautés qui fabriquent la ville en regardant dans l'espace urbain comment s'intègrent les migrations. Le programme Babels regroupe ainsi deux approches disciplinaires qui avaient tendance à s'ignorer jusque-là pour se retrouver sur un questionnement commun, celui de la ville et des migrations ${ }^{2}$.

V. B. : Le programme Babels privilégie une approche locale, celle des villes et des espaces urbains, mais en les replaçant dans deux autres échelles nationales et européennes dans lesquelles les migrations s'inscrivent. Ce qu'il y a de nouveau dans cette approche, c'est de considérer les villes comme des unités d'analyse et de les mettre en regard avec l'échelle étatique puisque dans de nombreux pays européens, ce sont les États qui prennent les décisions en matière de circulations et d'attribution des statuts. C'est aussi de privilégier l'échelle européenne qui apporte un contexte sur les modalités de ces circulations selon la géographie où elles se déroulent, et qui dessine différents réseaux par lesquels les personnes vont déterminer leurs trajectoires individuelles ou collectives et leur installation dans les villes.

C. M. : À cet égard, la petite étude sur la petite ville frontière de Kirkenes entre le Danemark et la Norvège est révélatrice de la mobilisation des citoyens qui se sont réunis de part et d'autre de la frontière pour faire passer des migrants, dans un contexte qui n'est pas celui des points de tension de l'espace Schengen et où les questions d'hospitalité demeurent présentes. Ce programme Babels a ainsi voulu observer comment les frontières peuvent modifier les enjeux locaux dans des endroits où les réalités des migrations sont situées hors des zones brûlantes de passage comme la frontière à Vintimille par exemple. Et comment le contexte géographique des villes implique des choix politiques pour la régulation des statuts des étrangers, mais aussi suscite ou non l'engagement des citoyens sur les problématiques migratoires.

V. B. : L'échelle de la ville permet aussi de poser de manière globale deux thématiques: d'une part, la question de l'hospitalité est envisagée en termes de citoyenneté et de proximité et, d'autre part, la question de la frontière qui n'est plus posée dans ses effets les plus massifs (les murs, les barbelés, les contrôles) mais en relation avec la vie quotidienne des migrants et des citoyens ordinaires. Les expériences que les

2. Cette dualité des études migratoires est analysée par Camille Schmoll dans «Spatialités de la migration féminine en Europe du Sud. Une approche par le genre», habilitation à diriger les recherches, Université Paris-Didierot, juin 2017 
- Mobilier urbain 1. (c) Paul Tailheuret. 
migrants font au quotidien des frontières dans les villes peuvent interpeller les citoyens sur la nature de l'État, même s'ils ne sont pas confrontés eux-mêmes à ces frontières de manière brutale, et donc susciter éventuellement de leur part des gestes de solidarité avec les migrants.

\section{$H \& M$ : Est-ce pertinent de distinguer les jeux d'acteurs territoriaux dans les métropoles entre les États, les élus municipaux, les associations et les collectifs divers et les habitants? Les logiques de ces acteurs sont-elles communes, concurrentes, en tensions ou en conflits selon les villes considérées?}

V. B. : D'une part, les acteurs - qu'ils soient municipaux, associatifs ou des citoyens - vont nécessairement s'inscrire dans le cadre de contraintes imposées par la politique étatique. Si on prend le cas de l'Allemagne, malgré les évolutions récentes, l'annonce de l'accueil des réfugiés par Angela Merkel au moment de ladite «crise migratoire» a instauré un climat qui n'était pas celui d'une confrontation avec les pouvoirs publics mais qui était plus favorable aux initiatives locales d'hospitalité envers les personnes migrantes et réfugiées. La manière dont les acteurs vont s'investir ou non dans des actions est aussi liée au contexte local et à l'histoire des villes. L'histoire des migrations intérieures est à ce titre très significative dans l'investissement des quartiers. À Berlin, dans certains quartiers, il y avait une longue tradition d'accueil des migrations turques ou de populations déplacées par les associations. C'est vrai également à Paris comme dans le XVIII ou le $\mathrm{XIX}$ arrondissement qui sont des quartiers de migrations et qui ont adopté quantité d'initiatives en direction des migrants par le passé. Les habitants veulent inscrire cette période dans une histoire migratoire depuis le $\mathrm{XIX}$ siècle. C'est tout l'intérêt des analyses micro-locales que de faire prendre conscience de la diversité des jeux d'acteurs qui se déroulent, dans les quartiers, à l'égard des migrations. Cela permet de comprendre comment se mettent concrètement en pratique les actions d'hospitalité et ce qu'elles signifient pour les populations locales. La compassion ou l'empathie avec son prochain ne suffisent pas nécessairement pour développer des interactions profitables et sereines.

C. M. : Dans ce programme de recherche, la nouveauté se situe aussi dans l'émergence de la ville comme acteur des politiques migratoires qui relevaient jusqu'alors d'une prérogative exclusive de l'État, avec un jeu entre l'échelle européenne et régionale. À cette échelle de la ville, de nouvelles associations, ou de nouvelles formes associatives sont également apparues, comme au
Danemark où des formes de politisation des populations - d'ailleurs à la limite de la dépolitisation - reposent les enjeux politiques en des termes humanitaires. Les nouvelles formes d'engagement citoyen dans des tissus associatifs parfois extraordinairement denses et dynamiques comme en France butent sur une absence

\section{Ce climat de confrontation est une des caractéristiques en France d'une politique publique de l'urgence que l'on ne retrouve pas forcément dans d'autres pays.}

de concertation et coordination entre les associations et les pouvoirs publics. Ce climat de confrontation est une des caractéristiques en France d'une politique publique de l'urgence que l'on ne retrouve pas forcément dans d'autres pays. Albert Hirschman dans son ouvrage Exit, voice and loyalty décrit trois façons de se positionner vis-à-vis du pouvoir en situation de crise: l'abandon, la confrontation, la loyauté. Or ses observations soulignent le lien qui existe entre voice et loyalty: ceux qui s'opposent à l'institution le font par loyauté aux valeurs de celle-ci qu'ils estiment trahies. De même, les citoyens les plus opposés aux pratiques des mairies, des États ou de l'Union Européenne le sont souvent par attachement à la citoyenneté, à l'État de droit, à l'Europe.

Les médias qui ne retranscrivent pas la dimension complexe des mobilisations citoyennes font le jeu des binarités et ne représentent les protestations que sous le prisme du trouble à l'ordre public. Ne pas expliquer cette complexité, c'est faire le jeu de tous les calculs politiques qui exploitent la question migratoire à des fins électoraliste - ce qui est la pratique récurrente de notre vie politique tout de même. À ce propos, l'actualité européenne évolue très rapidement. Publiant notre ouvrage début 2018, nous avons vu l'extrême droite s'imposer comme acteur politique en Allemagne, en Italie ou en Autriche avant même que l'ouvrage ne sorte de chez l'imprimeur ou peu de temps après. Cette actualité nécessiterait des mises à jour permanentes sur les jeux politico-électoralistes. Mais ce qui est analysé sur la tolérance dans les quartiers d'implantation à l'égard des centres d'accueil des réfugiés n'est pas pour autant invalidé. Si la ville de Grande-Synthe avait été inclue dans notre panel, les travaux seraient devenus des documents d'archives puisque le camp et les entrepôts dans lesquels étaient conservées les aides ont brûlé, les relations entre l'État, la ville et les associations ont empiré de manière drastique. 
Les études constituent des formes d'analyse du réel qui font comprendre de quelle manière et à quelle vitesse les situations locales peuvent changer.

\section{H\&M: Le programme de recherche Babels fournit-il un recueil de "bonnes pratiques » qui pourraient être transposables dans d'autres villes ou du moins influencer les programmes des politiques municipales ou étatiques?}

C. M.: Les travaux du programme Babels sont davantage une invitation aux débats à différentes échelles qu'un recueil de «bonnes pratiques». La question des bonnes pratiques est compliquée parce qu'il y a un niveau où les solutions sont relativement simples, comme dans le domaine de la justice ou de l'école, où l'enjeu est d'abord celui des moyens, insuffisants. Sur la question migratoire, il faut créer de la concertation entre les associations et les pouvoirs publics et y mettre les moyens budgétaires (qui aujourd'hui existent mais sont déployés dans une répression absurde, comme l'illustre la destruction de Grande-Synthe). Les associations le disent depuis plus longtemps que les sociologues et avec plus de précision et de force. Ces solutions simples ne sont pas à ériger en bonnes pratiques par des experts qui sont moins au fait des réalités locales que ne le sont les associations placées dans une situation à pallier les déficiences de l'État. Si les mairies et les préfectures n'arrivent pas à développer de la concertation, que peuvent faire les associations? Dans le contexte actuel où le problème est avant tout celui d'une lecture politique de la question migratoire en termes de crise, d'invasion etc., et où tout découle de cette problématisation, il peut être dangereux de traquer des initiatives (toujours palliatives) pour les hisser au rang de solutions exemplaires. Les solutions ne peuvent être pensées et posées qu'à un autre niveau, en termes de volonté politique. À cet égard, le contre-exemple d'Istanbul est intéressant: l'hospitalité y fonctionne grâce à la prise en charge des réfugiés syriens par des associations qui ont reçu de la part de l'État une sorte de délégation tacite. Mais, dans le contexte de la Turquie d'aujourd'hui, les associations islamistes liées au pouvoir renforcent l'enracinement des populations au parti d'Erdogan et le retrait des pouvoirs publics sert politiquement au parti majoritaire. Est-ce que c'est une bonne pratique et a-t-on envie de s'en inspirer?

H\&M: «Ce que les migrants font aux villes? " Cette interrogation portée par le dernière chapitre de votre livre est-elle moins souvent développée dans les programmes de recherche? Comment les acteurs peuvent-ils dépasser les situations d'urgence qui répondent aux politiques sécuritaires et restrictives et créent une pression constante autour de l'arrivée des migrants?

C. M. : Il ne faut pas oublier que la temporalité de notre recherche est très courte («Flash asile» dit bien son nom). Le programme a voulu souligner la dimension importante de gestion de l'urgence, la façon de l'État de se mettre dans l'urgence et de créer de l'urgence. L'urgence permet aussi aux chercheurs d'en analyser les ressorts et les conséquences sur les migrations et les populations urbaines. Les politiques d'immigration revendiquent une situation d'urgence mais laissent souvent les situations se dégrader afin de pouvoir les qualifier ensuite d'urgentes de manière plus crédible. Or la question de ce que font les migrants sur nos villes ne peut se traiter que sur du plus long terme,

\section{Sur la question migratoire, il faut créer de la concertation entre les associations et les pouvoirs publics et y mettre les moyens budgétaires (qui aujourd'hui existent mais sont déployés dans une répression absurde, comme l'illustre la destruction de Grande-Synthe).}

notamment sur plusieurs générations. Les études en sociologie travaillent depuis longtemps sur les effets des migrations sur les villes mais pour le programme Babels, les deux années n'étaient pas suffisantes.

V. B.: C'est pour cela que cette question a plutôt été traitée en conclusion du livre car elle est plus difficile à aborder et à mesurer, durant une période où les législations sur les migrations et l'asile, mais aussi le règlement de Dublin étaient constamment en train de changer. C'était d'ailleurs intéressant de voir comment les acteurs étaient constamment en train de repenser leur manière de voir les migrations, pour conseiller les personnes en situation de migration. On a tenu compte de l'importance du localisme dans les mesures prises en France par les préfectures en matière d'expulsion, d'assignation à résidence ou de traitement des dossiers administratifs, etc. On a essayé de montrer comment se crée cette situation d'urgence dans la gestion des migrations: la situation des personnes en demande d'asile, et même celles qui sont en amont des demandes d'asile, participe à la création des campements dans les villes à partir de leur exclusion des démarches administratives. 
- Mobilier urbain 2. (c) Paul Tailheuret.

C. M.: L'une des spécificités de la France, très visible à Paris, c'est la façon dont la question de l'hébergement est rattachée à celle du statut administratif. Réfléchir sur les effets de cette corrélation permet d'identifier la nouvelle figure du «sans-abris demandeur d'asile» ou du «sans-abris-migrant» et de comprendre les perceptions et les rejets qui se dessinent dans les populations parisiennes. L'étude a identifié le rôle de la préfecture à la fois comme manifestation de l'État, comme échelle d'intervention et comme de forme de décision administrative, dans son opacité, ses formes de hiérarchisation, ses collaborations avec les associations. Cet acteur-préfecture ne se retrouve peut-être pas dans d'autres pays européens. La troisième particularité française est de voir comment la gestion de la crise migratoire devient une politique publique.

H\&M : Les démarches d'enquête et de diffusion des résultats adoptées par le programme Babels visent-elles aussi à mettre la recherche au service de l'action publique? Quels sont les enjeux en termes de transmission des mémoires des luttes en 


\section{faveur des droits des migrants et de l'hospitalité? Sont-elles aussi un vecteur pour développer plus de citoyenneté et d'engagement à l'échelle locale?}

C. M.: L'intérêt des ateliers d'anthropologie publique est de ne pas s'inscrire dans le schéma classique de la diffusion des travaux vers la société plus large. Dans ce programme de recherche, en amont de l'écriture des ouvrages, les ateliers ont permis de poser les idées, de les présenter et de les discuter par des échanges avec les acteurs associatifs et autres. Les participants avaient plusieurs casquettes. Par exemple, le réseau Migreurope est composé de chercheurs militants qui transfèrent les problématiques d'un domaine à l'autre. En fait, ce sont souvent les questionnements des militants qui s'insèrent dans la recherche et y produisent un nouveau questionnement.

V. B.: L'anthropologie publique s'inscrit dans une tradition anglo-saxonne et permet ce jeu d'interactions entre les questionnements et la façon dont la recherche s'en saisit. Ce n'est donc pas une vulgarisation de la recherche mais une tentative de construire le savoir ensemble à partir des problématiques de terrain. En France, cette pratique n'est pas si fréquente parce que les frontières entre recherche et engagement sont mouvantes sur des enjeux politiquement sensibles. Ce programme Babels défend l'idée que la recherche ne peut être qu'engagée et ne peut pas avoir une position extérieure avec une vision neutre des situations. D'où l'idée d'une co-production du savoir avec l'ensemble des acteurs impliqués.

C. M.: La parution de l'ouvrage s'est faite au moment de l'organisation des États généraux des migrations qui va produire, en octobre 2018, une synthèse des «cahiers de doléances» rédigés dans 130 villes françaises, mobilisant des acteurs locaux pour décrire les expériences d'accompagnement des migrants. Notre livre a pu être présenté dans plusieurs villes (dont Dijon et Grenoble). L'ouvrage a donc servi d'outil dans les discussions en s'appuyant sur les situations locales observées en France et dans d'autres pays européens. Il a aussi participé à la formulation d'arguments pouvant réfuter certains mots d'ordre comme, par exemple, celui sur «l'appel d'air». Or tout l'échafaudage de la gestion de crise repose sur des fausses évidences qu'il faut arriver à déconstruire.

V. B.: Il faut trouver des formes d'expression que les gens puissent entendre ou lire facilement. Tous les arguments montrant les bénéfices concrets (y compris en termes économiques) de l'accueil des migrants ont été depuis des années construits et légitimés par des travaux scientifiques, mais personne ne lit ces études ni ne les utilise. L'enjeu est notamment de refuser l'articulation entre l'accueil des migrants et les poussées de l'extrême droite, car c'est le contraire qui s'est passé: ne pas démonter ces fausses évidences permet aux extrêmes politiques d'en profiter. Les travaux de terrain doivent être accompagnés par des débats, sinon ils glissent sur le discours sur les migrations qui est lissé par les médias et les partis politiques et patinent sur ce glacis médiatico-politique sans toucher les opinions.

\section{Tous les arguments montrant les bénéfices concrets (y compris en termes économiques) de l'accueil des migrants ont été depuis des années construits et légitimés par des travaux scientifiques, mais personne ne lit ces études ni ne les utilise.}

C. M.: Les personnes engagées lisent des études plus poussées et s'informent sur les réseaux sociaux pour mieux échanger entre elles. Camille Schmoll et d'autres chercheurs ont lancé cet été une tribune dans Le Monde pour relancer le débat sur la nécessité, pour les décideurs des politiques migratoires, de consulter des experts. Il y a un état de l'art sur les migrations accepté par la communauté scientifique sur l'inefficacité des politiques des frontières, sur l'ineptie de l'appel d'air (qui est une "fake news»), comme sur l'apport économiques des migrations. Néanmoins le transfert du savoir vers le monde médiatico-politique ne se fait pas. 